\title{
INNOVATION THROUGH INTEGRATION
}

\section{A new initiative could provide the pharmaceutical industry with a model for how different research skills in a common field can be integrated effectively}

There is general agreement in the pharmaceutical industry that the disciplines that make up each stage of the drug discovery and development process are becoming increasingly specialized. As a consequence, so are the researchers who are being trained in these areas - both in academia and industry. But there is also a general feeling that industry should move away from this 'functional specialization' model and towards a more integrative model, in which researchers can apply their skills to several parts of the development pipeline.

Although the will might be there, the industry shows little sign of implementing any sea change in its recruitment policies, still preferring to recruit the best scientists in specialized fields. But change is beginning to occur in some areas. In the United Kingdom, for instance, the Wellcome Trust has adopted an initiative on Integrative Animal and Human Physiology, in which it has funded grants designed to "promote research exploring the relationship between genomic information and physiological mechanisms in humans and animals."

One of the initiatives that the Trust has funded, which is based at the University of Oxford, could provide a model for how different research areas in a common field can be integrated effectively. The initiative, called OXION (BOX 1), received a grant of $£ 4.8$ million ( $\sim$ US $\$ 7.5$ million) to focus on ion channels, a group of 250-300 membrane pores that orchestrate electrical signals through brain, heart and muscle cells, and which, after G-protein-coupled receptors, represent the biggest target for existing therapies.

The initiative has been set up to take advantage of the fact that world-class research in many different areas of ion channels is carried out at Oxford (and also the departments in Cambridge and London that are part of the initiative). However, researchers trained through the initiative will not receive a traditional apprenticeship. The aim is to train graduates and postgraduates to a high level in a whole range of techniques as a way of curtailing the tendency of researchers to focus too much on individual subjects. For example, many in the postgenomic age understand how to identify candidate genes for a certain disorder, but applying this to understanding how the gene product functions in the whole biological system is left to others. Providing an integrated research programme and training will allow researchers to see the wood, as well as the trees.

The graduate studentship entails one year of training in all disciplines on offer (genetics, functional genomics, neuroanatomy, neurophysiology, ion channel structure and function, and human neurological disorders), through a combination of lectures, practical courses and two 4-month research projects, which are spent in different laboratories. Years 2-4 are devoted to a multidisciplinary doctoral research project. This project could, for example, involve the molecular modelling of acetylcholine receptors, looking at the effect of mutations in acetylcholine receptor genes in heterologous systems, assessing the behaviour of model organisms (such as mice and worms) carrying mutant receptors, looking at proteins that complex with the receptor and studying human diseases caused by mutant acetylcholine receptors or autoantibodies to the receptor. The doctoral projects therefore span at least two laboratories. There are also training fellowships, in which postdoctoral researchers carry out their own research, as well as learning new approaches themselves - partly through helping to train the graduate students in a 'training-by-teaching' approach.

The OXION initiative admitted its first training fellow last year and plans to officially launch at the beginning of the coming academic year. During the next five years, OXION will fund ten postdoctoral studentships and six training fellowships. For postgraduate positions, candidates can come from a range of life-science backgrounds as they are trained in-house; postdoctoral positions are more likely to be offered to people from an appropriate background, who should be able to 'hit the ground running.

Many other academic institutions have the facilities for similar initiatives, although setting these up would of course be dependent on securing a similar level of funding. However, one place where the facilities and finance to adopt such a model are available under one roof is in pharmaceutical companies. At a time when building increased levels of integration is becoming an important strategy in driving R\&D forward, the success of the OXION initiative will be keenly followed.

Simon Frantz

\section{Box 1 | Further information}

OXION http://oxion.physiol.ox.ac.uk/

Simon Frantz, Associate Editor Nature Reviews Drug Discovery. e-mail:s.frantz@nature.com

OXION has been established by a consortium of scientists at the University of Oxford, in collaboration with groups at Cambridge University and the Institute of Neurology and Neurological Disease, London. The Director of OXION is Frances Ashcroft and the Director of Training is Nick Rawlins. The website contains details of the initiative, including research, training and positions available. 